\title{
Preface - Special Issue to Celebrate the 30th Anniversary of Journal of Systems Science and Complexity
}

\author{
GAO Xiao-Shan $\cdot$ CHEN Jie $\cdot$ SHAO Jun $\cdot$ WANG Shouyang
}

DOI: $10.1007 / \mathrm{s} 11424-017-6000-4$

Received: 10 September 2016

(C)The Editorial Office of JSSC \& Springer-Verlag Berlin Heidelberg 2017

This special issue is dedicated to the 30th anniversary of Journal of Systems Science and Complexity (JSSC) which is under the auspices of the Institute of Systems Science, Chinese Academy of Sciences.

Institute of Systems Science, Chinese Academy of Sciences was established in 1979 by scientists Chao-Chih Kwan, Wen-Tsun Wu, Guo-Zhi Xu. In 1981, the Journal of Systems Science and Mathematical Sciences (JSSMS) was founded as the institutional journal of Institute of Systems Science, which was published by Science Press domestically. The journal focused on "research papers that are original in theory and method in the fields of systems science and mathematical sciences and technical reports that are original in solving practical problems." JSSMS publishes papers both in Chinese and English and the papers were from "research personnel of Institute of Systems Science and a small number of invited papers from other institutions as well."

In order to increase its international influence, an international journal Systems Science and Mathematical Sciences (SSMS) was founded as the English version of JSSMS in 1988, which accepts submissions from all over the world. The founding Editor-in-Chief of SSMS is Professor Han-Fu Chen. The scope of the journal includes "systems theory, system modeling, systems control, systems analysis, systems management, systems engineering, information processing, mathematical physics, mathematical statistics, computer mathematics, combinatorics and optimization, operations research, applied functional analysis and relevant topics

GAO Xiao-Shan

Academy of Mathematics and Systems Science, Chinese Academy of Sciences, Beijing 100190, China.

Email: xgao@mmrc.iss.ac.cn.

CHEN Jie

Key Laboratory of Intelligent Control and Design of Complex Systems, Beijing Institute of Technology, Beijing 100081, China. Email: chenjie@bit.edu.cn.

SHAO Jun

Department of Statistics, University of Wisconsin-Madison, Madison, USA. Email: shao@stat.wisc.edu. WANG Shouyang

Academy of Mathematics and Systems Science, Chinese Academy of Sciences, Beijing 100190, China.

Email: sywang@amss.ac.cn. 
in modern mathematics." SSMS was published by Science Press in China and Allerton Press internationally.

In 2001, to further highlight the field of systems science and the great prospect of the emerging complexity science, the journal's name was changed to Journal of Systems Science and Complexity (JSSC). The founding Editor-in-Chief of JSSC is Professor Lei Guo. JSSC focuses on "systems theory, systems engineering, systems control, systems modeling and statistics, systems management, intelligent systems, complex systems, and complexity science for the best benefit of the scientific community in systems science, and for the promotion of developments in the emerging complexity science." The current focus of JSSC is "complex systems and control, operations research and economic analysis, statistics and data science, computer mathematics." JSSC has been published by Springer since 2006 and has been indexed by SCI and EI since 2007.

JSSC has become an important publication platform for the community of systems science and related areas and has formed the unique feature of publishing original results in the multidisciplinary borders among complex systems, systems control, economic system analysis, data science, and computer mathematics. Some of the pioneering work in these interdisciplinary fields appeared first in JSSC. For instance, the first systematic presentation for the theory of Mathematics Mechanization was published in the following paper ${ }^{[1]}$ :

Wen-Tsun Wu, Basic Principles of Mechanical Theorem Proving in Elementary Geometries, Journal of Systems Science \& Mathematical Sciences, 1984, 4(3): 207-235.

This highly influential paper was re-published in Journal of Automated Reasoning in 1986 due to great demands by researchers outside China and the fact the paper was not widely available at that time. Due to his contribution to mathematics mechanization and topology, Wen-Tsun Wu was awarded the first State Supreme Science and Technology Award of China in 2000 and the Shaw Prize for Mathematics in 2006. Another landmark paper published by JSSC is ${ }^{[2]}$

John H. Holland, Studying Complex Adaptive Systems, Journal of Systems Science \& Complexity, 2006, 19(1): 1-8.

In this paper, Professor Holland introduced the general properties of Complex Adaptive System and proposed major challenges for studying them. Complex adaptive systems are now one of the major frameworks for studying complex systems. The third example of pioneering papers published by JSSC is ${ }^{[3]}$

Gongguo Tang and Lei Guo, Convergence of a Class of Multi-agent Systems in Probabilistic Framework, Journal of Systems Science \& Complexity, 2007, 20(2): 173-197.

This paper gave the first proof for synchronization of a basic class of multi-agent systems by establishing the connectivity of the associated dynamic graphs, which formed the basis of a subsequent work by Chen, Liu and Guo (2012) that was later selected and republished by SIAM Review as a SIGEST paper in 2014.

Through the past thirty years, JSSC has been receiving support from authors and readers in the field of systems science and related areas, members of the editorial boards, funding agencies, publishers, and the sponsoring institution. We would like to take this opportunity to show our appreciation for their long term support of JSSC.

Springer 
This special issue was organized by the editorial board of JSSC. The papers published in this special issue ${ }^{[4-17]}$ are high quality contribution to the fields of complex network, systems control, statistics and data science, and computer mathematics. We would like to express our gratitude to all the authors for their contributions and support.

\section{References}

[1] Wu W T, Basic principles of mechanical theorem proving in elementary geometries, Journal of Systems Science \& Mathematical Sciences, 1984, 4(3): 207-235.

[2] Holland J H, Studying complex adaptive systems, Journal of Systems Science \& Complexity, 2006, 19(1): 1-8.

[3] Tang G G and Guo L, Convergence of a class of multi-agent systems in probabilistic framework, Journal of Systems Science \& Complexity, 2007, 20(2): 173-197.

[4] Li Y, Mu Y F, Yuan S, et al., The game theoretical approach for multi-phase complex systems in chemical engineering, Journal of Systems Science \& Complexity, 2017, 30(1): 4-19.

[5] Hu Y Q, Fan Y, and Di Z R, Orientation in social networks, Journal of Systems Science \& Complexity, 2017, 30(1): 20-29.

[6] Liu X Y, Sun J, Dou L H, et al., Leader-following consensus for discrete-time multi-agent systems with parameter uncertainties based on the event-triggered strategy, Journal of Systems Science \& Complexity, 2017, 30(1): 30-45.

[7] Wen G H, Yu W W, Yu X H, et al., Complex cyber-physical networks: From cybersecurity to security control, Journal of Systems Science \& Complexity, 2017, 30(1): 46-67.

[8] Yan Y M and Huang J, Robust output regulation problem for discrete-time linear systems with both input and communication delays, Journal of Systems Science \& Complexity, 2017, 30(1): 68-85.

[9] Yang Y C and Hu X M, Optimal control using microscopic models for a pollutant elimination problem, Journal of Systems Science \& Complexity, 2017, 30(1): 86-100.

[10] Hu X N, Duan X G, Pan D D, et al., A model-embedded trend test with incorporating HardyWeinberg equilibrium information, Journal of Systems Science \& Complexity, 2017, 30(1): 101110.

[11] Xia Q and Dong Y X, On a new hybrid estimator for the central mean space, Journal of Systems Science \& Complexity, 2017, 30(1): 111-121.

[12] Zhang Y Q and Tang N S, Bayesian empirical likelihood estimation of quantile structural equation models, Journal of Systems Science \& Complexity, 2017, 30(1): 122-138.

[13] Zhao J W and Shao J, Approximate conditional likelihood for generalized linear models with general missing data mechanism, Journal of Systems Science \& Complexity, 2017, 30(1): 139153.

[14] Chen S S and Kauers M, Some open problems related to creative telescoping, Journal of Systems Science \& Complexity, 2017, 30(1): 154-172.

[15] Gao X S, Huang Z, Wang J, et al., Toric difference variety, Journal of Systems Science \& Complexity, 2017, 30(1): 173-195.

[16] Kapur D, Comprehensive Gröbner basis theory for a parametric polynomial ideal and the associated completion algorithm, Journal of Systems Science \& Complexity, 2017, 30(1): 196-233.

[17] Wang Q Y, Li Y J, Xia B C, et al., Generating semi-algebraic invariants for non-autonomous polynomial hybrid systems, Journal of Systems Science \& Complexity, 2017, 30(1): 234-252. 